\title{
Miniaturized Meander Slot Antenna Tor RFID TAG with Dielectric Resonator at $60 \mathrm{Ghz}$
}

\author{
JMAL Sabri \\ Dept. of Physics, \\ El Manar University, Faculty of \\ Sciences, \\ Tunisia
}

\author{
NECIBI Omrane \\ UR of Circuits and Elec. Sys. HF, \\ Dept. of Physics, \\ El Manar University, Faculty of \\ Sciences, \\ Tunisia
}

\author{
TAGHOUTI Hichem \\ Departement of physics, chimical \\ and processes Engineering \\ University of Carthage
}

\author{
MAMI Abdelkader \\ Dept. of Physics, \\ El Manar University, Faculty of Sciences, \\ Tunisia
}

\author{
GHARSALLAH Ali \\ UR of Circuits and Elec. Sys. HF, Dept. of Physics, \\ El Manar University, Faculty of Sciences, \\ Tunisia
}

\begin{abstract}
Day after day, recent advances in millimeter wave communications have called for the development of compact and efficient antennas. Furthermore, the greatest challenges in this area is to get a good performance and a miniaturized antenna. In addition, the design of antenna, in the Silicon technology, is one of the key challenges. In this way, this work will focus on the design of the free $60 \mathrm{GHz}$ band, high gain and high efficiency onchip antenna meandering slots for transponder Radio Frequency Identification (RFID). Further, the stacked dielectric resonators (DRS) will be arranged above the power element of on-chip antenna with an excitation of coplanar waveguide (CPW). We will use the Scattering Bond-Graph formalism like a new technique to design these proposed antennas and we will use the microwave Studio CST software simulation to validate the results. We have miniaturized the proposed antenna after having such a number of iteration and by applying the Bond Graph methodology, and the size of the antenna is about $1.2 * 1.1 \mathrm{~mm}^{2}$.
\end{abstract}

Keywords-RFID TAG; Millimeter Wave Identification; Meander Slot Antenna; Dielectric Resonator Antenna (DRA); Onchip Antenna; Silicon; Scattering Matrix; Bond-Graph; Scattering Bond-Graph

\section{INTRODUCTION}

In the last few years, radio frequency identification (RFID) has become more and more popular in numerous applications, such as logistics, supply chains management, assets follow-up (active persons), and vehicles positioning [1]. Among a variety of RFID systems using radio frequencies, an RFID Ultra Height Frequency (UHF) system drew a lot of attention because of its numerous advantages, such as the cost, the size, and the long-range reading [2].

Millimeter Wave Identification (MMID) modernizes the RFID system to millimeter waves [3]. The higher frequency systems have several advantages: First, smaller antennas allow us to create miniaturized transponders. Second, antenna arrays can achieve a narrow beam for the reader antennas, which allow a well-organized transponder localization. Third, the regulations of relaxed radio at $60 \mathrm{GHz}$ allow great data communication rate.

The most obvious advantage of the technology of $60 \mathrm{GHz}$ is its strong capacity in terms of debit. Indeed, the Shannon theorem (1) shows that the capacity $\mathrm{C}$ of a communication channel $(\mathrm{C}=$ Max debit. $)$ increases linearly with the bandwidth BW, while it increases following a logarithmic law according to the report signal with noise SNR:

$$
C=B W \cdot \log _{2}(1+S N R)
$$

Thus, this relation leads us to conclude that to increase the debit of a radio channel, it is necessary to widen its bandwidth, rather than improve its report signal with noise. At these frequencies, antenna with dielectric resonator is the most voluminous component and its miniaturization constitutes one of the most important current challenges for the designers of communicating objects [4]. The use of dielectric resonators in the design of antennas for RFID tags in the EHF band has never been made. However, the miniaturization of antennas is usually accompanied by deterioration of its efficiency and bandwidth, because materials with high permittivity are generally used [5]. Therefore, miniature structures designed must present a compromise between bandwidth efficiency and physical size. Our objective is to conceive a structure of antenna with miniature dielectric resonators for RFID TAG in the $\mathrm{V}$ band $(60 \mathrm{GHz})$ with correct performances.

On the other hand, the Bond Graph approach has showed [6], together with the Scattering formalism [7], its usefulness in studying and understanding the antennas [8].

A new way of applying the scattering bond graph technique will be used in this article. It is to show that the scattering bond graph methodology can serve as a new miniaturization approach to the design of RFID TAG in the V band.

In this paper, we focus only on one component of the MMID system, namely, the design of a very small antenna for an RFID TAG with a silicon technology. 
In addition, in this work we are going to study the design and the simulation of an antenna with dielectric resonator of rectangular shape for RFID TAG in the EHF band. Then we are making a comparative study in terms of gain and volume of our structure with an UHF antenna of the trade and we finished our work with the validation of the simulation results by the Scattering Bond Graph methodology.

\section{THE BASIC 60GHZ RFID ANTENNA}

In radio communication, antenna is a key element. We all know that antenna recovers the power and data signal traveling through the air. The quality and performance of the antenna are involved in the quality of wireless communication.

\section{A. First Iteration}

The configuration of the proposed antenna is shown in Figure 1.

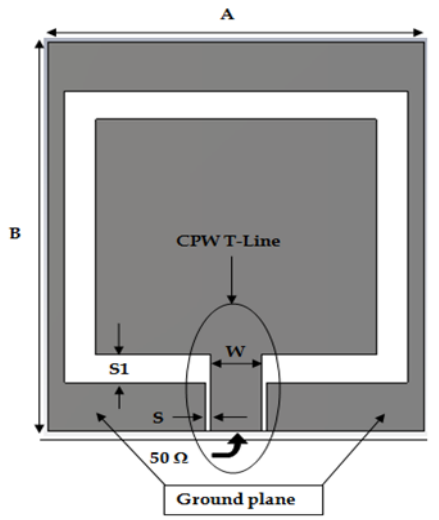

Fig. 1. This 3D on-chip antenna-top-sectional view of the feeding structure ( $\mathrm{x}-\mathrm{y}$ plane), where $\mathrm{A}=1.77, \mathrm{~B}=2, \mathrm{~W}=0.24, \mathrm{~S}=0.025, \mathrm{~S} 1=0.145 ;$ ( all dimensions in $\mathrm{mm}$ )

The antenna structure design is based on the optimal length of the radial stub protrusion, which is in the range of 0.45-0.6 times the slot's width. Further, we have achieved the impedance matching by increasing the width of printed stub. We have also noted that the edge treatment of the stub has an impact on the bandwidth improvement. In addition, we have represented the slot region between the antenna input, the edge of the patch and the distal slot region, by Bond Graph elements by lumped admittances (capacitance and inductance). Moreover, this physical interpretation has been deduced by using the Bond Graph model shown in Figure 2.

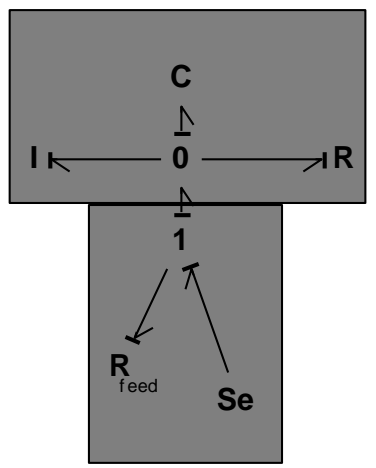

Fig. 2. 3D on-chip antenna-top-sectional Bond Graph model
Where the equivalent model of patch antenna is shown on the resistance $\mathrm{R}$, inductance $\mathrm{I}$ and capacitance $\mathrm{C}$ interconnected by a " 0 -junction". A resistance $\mathrm{R}_{\text {feed }}$ and an effort source $\mathrm{S}_{\mathrm{e}}$ modulate the excitation and the $\mathrm{CPW}$ line interconnected by "1-junction".

Thus, for the selected size of ground plane, we have a good input impedance-matching can be obtained and it is verified by our Bond-Graph methodology.

\section{B. Second Iteration}

The proposed antenna consists on a modulated loop element printed on the Rogers substrate. A detailed on-chip antenna is presented in Figure 3 with S1 is the rings width, S is the gap between the rings and $\mathrm{W}$ is the gap on both end of the rings. A coplanar waveguide (CPW) and a slot ring create the feeding structure [10]. It consists of a silicon based onchip feeding structure.

The configuration of the proposed meander slot-based RFID loop antenna is shown in Figure 3.

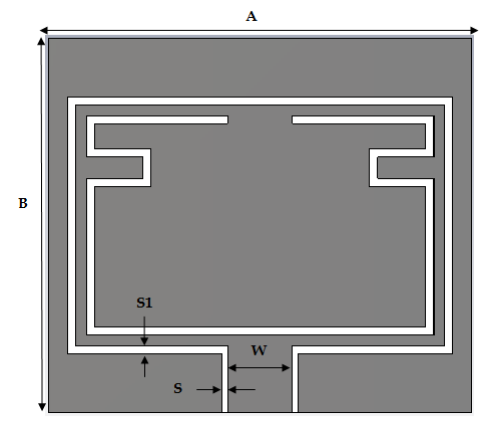

Fig. 3. 3D on-chip antenna-top-sectional view of the feeding structure (x-y plane), where $\mathrm{A}=1.57, \mathrm{~B}=1.4, \mathrm{~W}=0.24, \mathrm{~S}=0.02, \mathrm{~S} 1=0.03$; (all dimensions in $\mathrm{mm}$ )

\section{Simulation Results}

This section presents the simulated results of the meander slot antenna. We use the scattering bond graph methodologies [11] and we use the CST Microwave studio software, for several performance parameters such as impedance bandwidth, and radiation patterns. For the scattering bond graph methodology, we take into account the equations demonstrated in the above work [12].

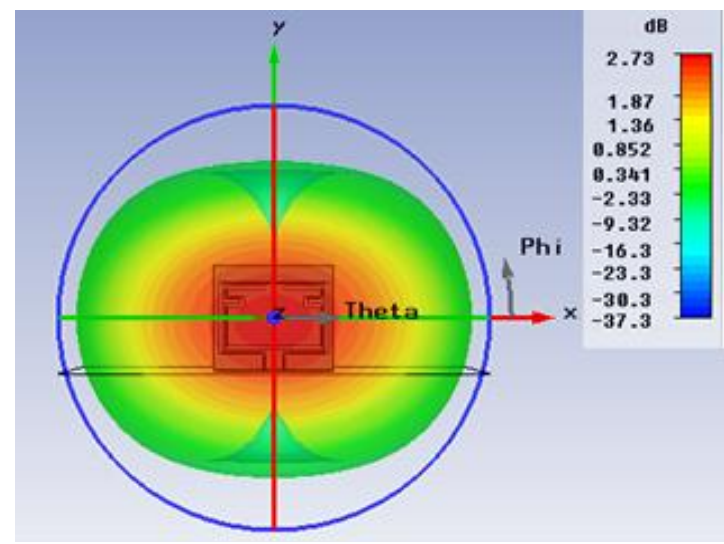

Fig. 4. Simulated gain of proposed antenna simulated by CST 
We note that structures present important parameters, as shown in the simulation results: From the reflection coefficient $\mathrm{S} 11$, the resonance frequency is about $60 \mathrm{GHz}$ for the two iterations. According to the far field, the two On-Chipantennas present an acceptable gain but it decrease from $3.02 \mathrm{~dB}$ to $2.73 \mathrm{~dB}$.

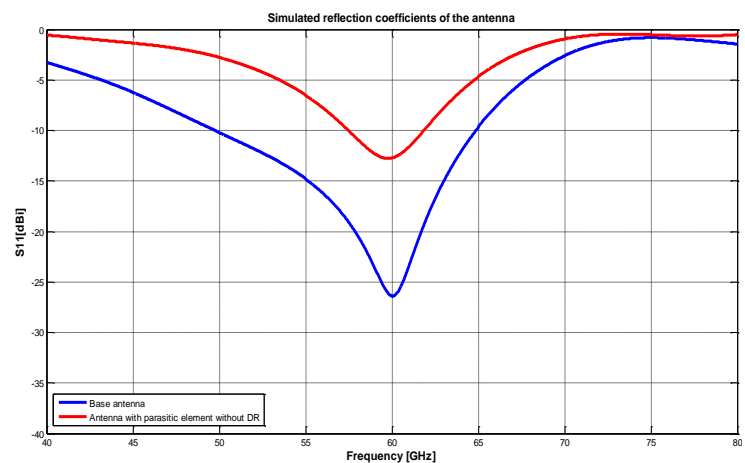

Fig. 5. Simulated reflection coefficient for the On-Chip antenna by Bond graph methodology

\section{Above IC}

To improve the performance of antennas on silicon, hybrid technology or "above IC" is proposed, which allow the optimization of the radiation efficiency by promoting the coupling between guided incident modes and radiated continuous modes.

Interests in silicon-based wireless system-on-chip (SOC) applications have become pervasive because of the advancement of its low-cost technology [13]. On-chip antennas eliminate the need for external off-chip connection and packaging process, which would incur a gain loss and overall size increase [14].

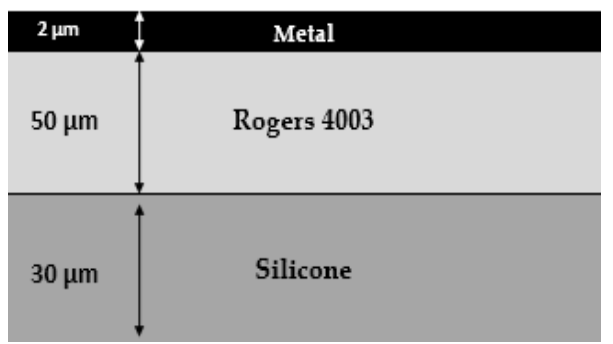

Fig. 6. Geometry of the proposed antenna (Button view)

We take into consideration the figure 6 shown blow we can conclude the bond graph model shown on the figure 7.

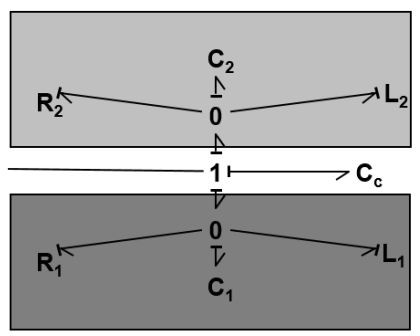

Fig. 7. Causal Bond graph model of proposed antenna

Where the values of the bond graph elements are deduced by the above work [15] That we have considered that any resonate element is considered a resistance $\mathrm{R}$ element, Capacitance element $\mathrm{C}$ and Inductance element I interconnected by 0 junction[16] .

To be sure, we can write the expression of the input impedance $Z_{\text {in }}[17]$

$$
Z_{i n}=\frac{R}{1+Q_{t}^{2}\left[\frac{f}{f_{r}}-\frac{f_{r}}{f}\right]^{2}}+j\left[X_{L}-\frac{R Q_{t}\left[\frac{f}{f_{r}}-\frac{f_{r}}{f}\right]}{1+Q_{t}^{2}\left[\frac{f}{f_{r}}-\frac{f_{r}}{f}\right]^{2}}\right]
$$

The resistive element $\mathbf{R}$ represented the losses of the antenna in its planar environment. $\mathbf{R}$ is calculated by the following [18].

$$
R=\frac{Q_{t} \cdot h}{\pi f_{r} \varepsilon_{d y n} \varepsilon_{0} W L} \cos ^{2}\left(\frac{\pi x_{0}}{L}\right)
$$

Where: $f_{r}$ : Resonant frequency; $Q_{t}$ : Quality factor; $\varepsilon_{d y n}$ : Dynamic permittivity; $x_{0}$ : Distance between the feeding point and the patch; L, W: Dimensions of the patch. h: Substrate height.

Electrical energy storage modeled by the capacitance C. It is represented by the following equation [19].

$$
C=\frac{Q_{t}}{2 \pi f_{r} R}
$$

To find $L$, we verify the relation represented in the following equation [20].

$$
L=\frac{R}{2 \pi f_{r} Q_{t}}
$$

The coupling between the two layers is reflected only by a capacitive element $\mathrm{C}_{\mathrm{c}}$ connected by 1 junction. The expression of this capacitance represented in the [21].

\section{The Proposed TAG ANTENNA WITH DIELECTRIC RES ONATOR}

This study consists in analyzing and modeling the geometry of simple antenna excited under the $T E_{\delta 11}^{X}$ mode. The radiating element is formed by the concentration of two materials with different permittivity: it is a vertical superposition of the portions in a concentric way. The purpose is to excite the two resonators at the same frequency to have single-band functionality.

\section{A. Rectangular Dielectric Resonator Antennas}

The rectangular shape of the Dielectric Resonator (DR) offers additional advantages compared with other geometrical forms. Indeed, it possesses (Fig.6) two independent parameters (w/h and w/a), which allow it, in one hand, to have two freedom degrees to determine a specific resonance frequency to a given value of dielectric permittivity. On the other hand, a good selection of the dimensions of the resonator, that helps us to avoid the problem of the degeneracy 
of modes, therefore an increase in cross-polarized antenna's implementation. The existence of the latter will degrade the antenna gain and will distort the radiation pattern [22]. Finally, the rectangular dielectric resonator antenna offers a greater flexibility in the optimization of the desired frequency band.

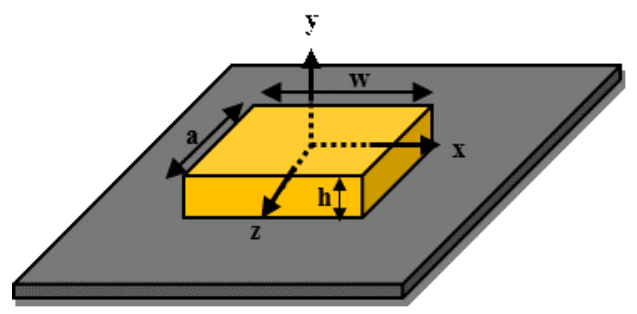

Fig. 8. Rectangular structures

After looking of this structure, we can deduce the bond graph model shown in Figure 9.

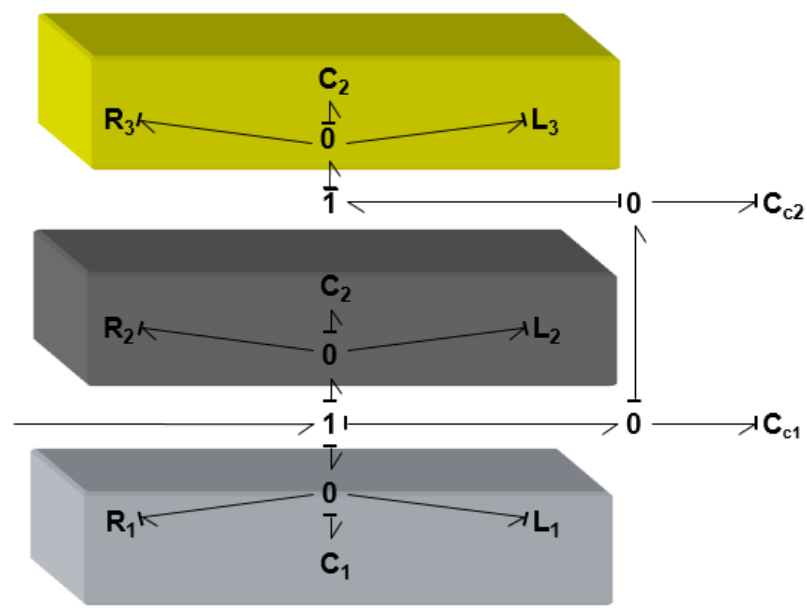

Fig. 9. Rectangular structures by Bond-Graph model

Analysis of the dielectric resonator antenna (DRA) can be made by considering the general classification methods proposed by Van Bladel for arbitrary forms of DR [23, 24]. According to Van Bladel, the modes of a dielectric resonator of high permittivity can be confined or not confined. For both types of modes, the electric component of the field, perpendicular to the dielectric/air interface, it must cancel what is explained in the following boundary condition

$$
\vec{n} \cdot \vec{E}=0
$$

With $\vec{E}$ the electric field vector and $\vec{n}$ the normal direction to the separation surface DR / air.

The last relation is one of two boundary conditions that must be satisfied. The second condition is given as below:

$$
\vec{n} \wedge \vec{H}=0
$$

Where $\vec{H}$ represents the magnetic field.

As regards the nature of material that composes the DR, relation (6) could be no longer satisfied. Therefore, by confined modes, we consider that all modes satisfy both two boundary conditions ((6) and (7)). Only dielectric symmetrical shapes such as sphere and cylinder cannot support them. On the other hand, the not confined modes satisfy only the equation (6) [25] and they can supported by any arbitrary shape of the DR. Consequently, the DR does not present symmetry of revolution; it only supports the not confined modes.

Marcatilli demonstrated that the existence of TM modes in a rectangular dielectric wave-guide is questionable [26], because they do not satisfy the boundary condition (6) consequently, for the analysis of modes in a rectangular dielectric resonator antenna, only $T E_{\delta 11}^{X}$ modes are considered.

The $T E_{\delta 11}^{X}$ mode of the DRA is particularly interesting for antenna applications because it has the lowest mode rank and has the lowest quality coefficient.

\section{B. Coupling Techniques}

In dielectric resonators antenna design, the coupling scheme has a very important role. Indeed, the coupling system and its location affect the performance of antenna in terms of bandwidth, radiation pattern and polarization. According to the basic electromagnetic theory and the reciprocity theorem of the Lorentz law [27], coupling $\mathrm{k}$ between the source (magnetic or electronic) and the fields inside the dielectric resonator can be determined by the following relationships:

$$
\begin{aligned}
& k \propto \int\left(\vec{E} \cdot \overrightarrow{J_{e}}\right) d V \\
& k \propto \int\left(\vec{H} \cdot \overrightarrow{\left.J_{m}\right)} d V\right.
\end{aligned}
$$

Where $\vec{E}$ and $\vec{H}$ are the vectors of the intensity of electric and magnetic field, $\vec{J}_{e}$ and $\vec{J}_{m}$ are the electric and magnetic currents, respectively.

To have a strong coupling between the source of electric current (magnetic) and dielectric resonator antenna, the source should be located where there is an important electric field (magnetic).

\section{Operating Principle of Series-Fed DRA Array}

Figure 10 shows the configuration of the proposed on-chip stacked DRA array. It consists on a feeding structure and one or more DRs. A dielectric resonator $\left(D_{1}\right)$ is mounted on the surface of the substrate, forming the traditional on-chip DRA investigated in [27] and [28].

A second dielectric resonator, $\mathrm{DR}_{2}$ is arranged above the antenna in the vertical direction (z-axis), forming a series-fed linear array.

In the proposed array design, all the DRs resonate at the same frequency $\mathrm{f}_{0}$ of the dominant mode $T E_{\delta 11}^{X}$. With the specified design frequency, the dimension of each DR having a dielectric constant of $\varepsilon_{\mathrm{r}}$ can be predicted using the traditional truncated dielectric waveguide model [29] or a simplified engineering formula presented in [30].

The operating mechanism of the proposed series-feed 
antenna array can be explained as follows: most of the energy is initially coupled to DR1 from the on-chip feeding structure. DR1with dominated mode ( $\left.T E_{\delta 11}^{X}\right)$ radiates with high efficiency there by acting as a traditional on-chip DRA. Then, part of the energy is serially coupled to the upper DRs $\left(D_{2}\right)$ to excite the mode.

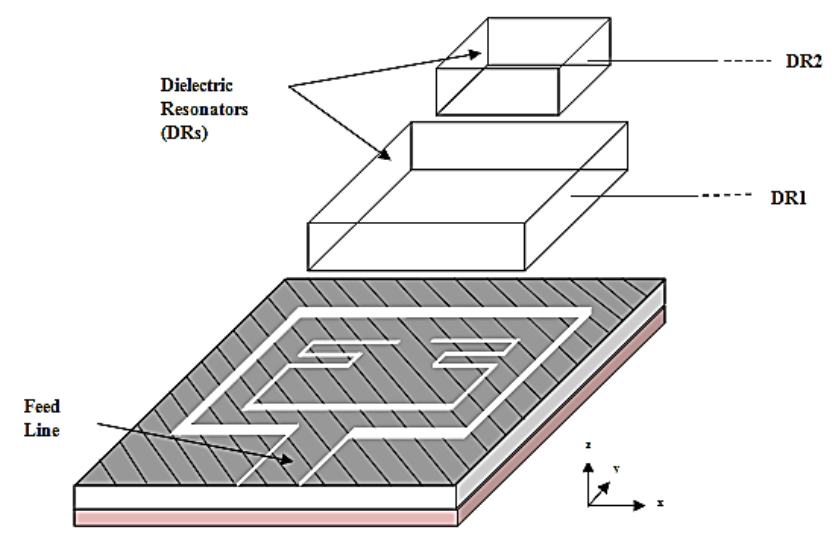

Fig. 10. On-chip stacked dielectric resonator antenna array: 3D structure of on-chip stacked dielectric resonators antenna array

The series coupling excitation leads to 180-phase difference of the electromagnetic (EM) waves between the two DRs.

\section{The Antenna Structure}

The proposed antenna is shown in the following figure.

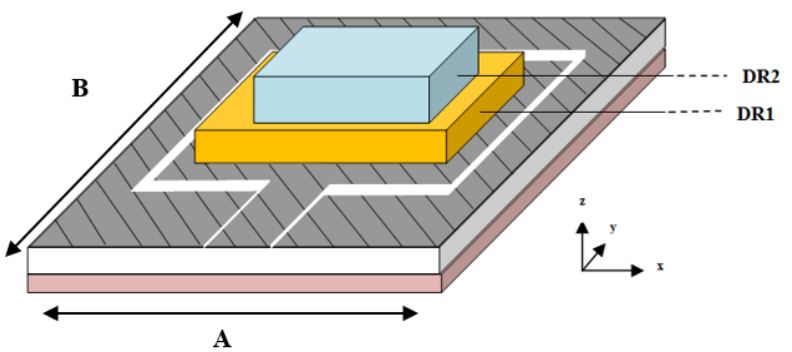

Fig. 11. 3D on-chip antenna with double dielectric resonators where $\mathrm{A}=1.2$, B (all dimensions in $\mathrm{mm}$ )

$=1.117$

The physical interpretation of the proposed antenna using the Scattering Bond Graph methodology give us the following Bond Graph model which is shown in the following figure.

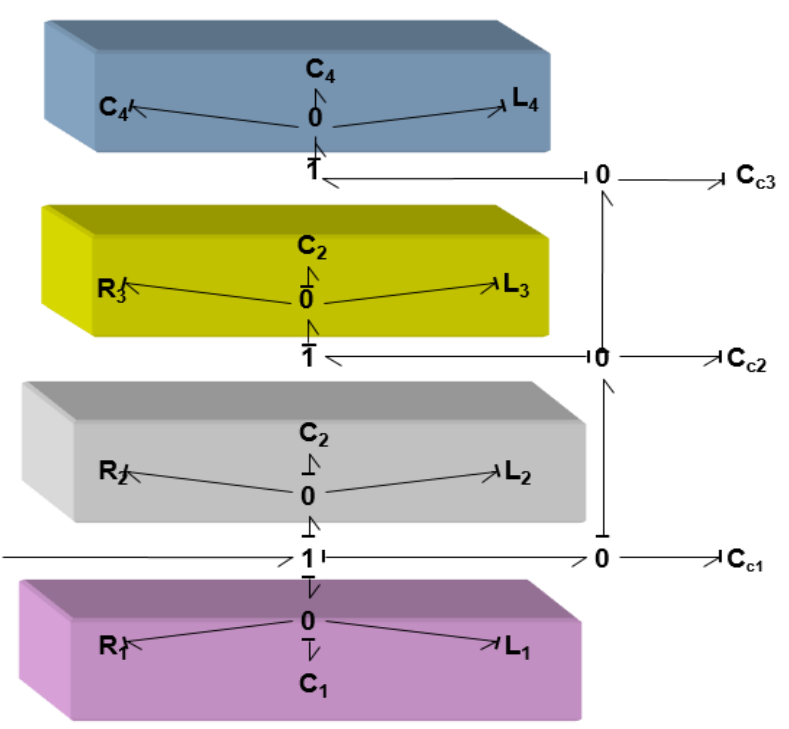

Fig. 12. On-chip antenna by bond graph methodology

A detailed double-DR arrangement of the on-chip antenna array is presented in Figure 11. It consists of a silicon based on-chip feeding structure and two DRs. A grounded coplanar waveguide (GCPW) and a meander slot create the feeding structure.

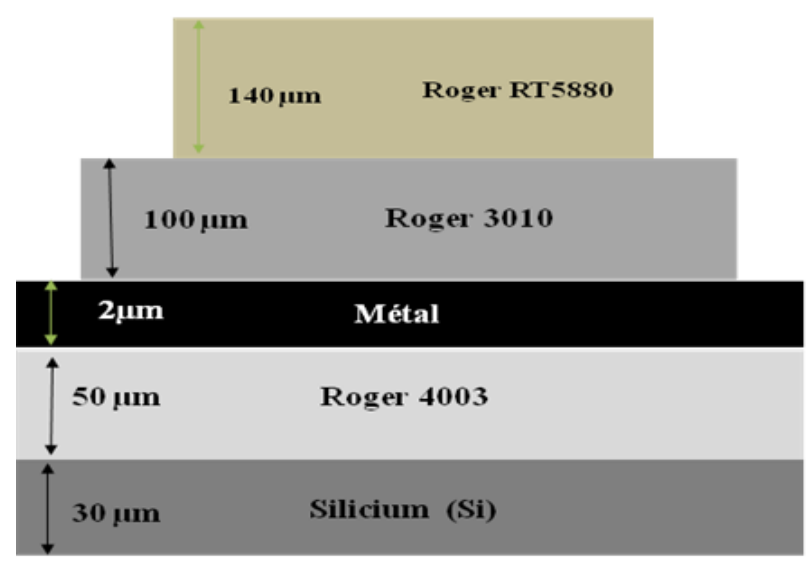

Fig. 13. Geometry of the proposed antenna with DRs (Button view)

The meander slot is implemented on the $2-\mu \mathrm{m}$ thick top metal. It is shielded by the bottom metal and connected to a $50 \Omega$ GCPW with signal line width of $\mathrm{w}=240 \mu \mathrm{m}$. The thickness of the Roger substrate is $50 \mu \mathrm{m}$. 
For this study, the dielectric constant, $\varepsilon_{\mathrm{r} 1}$ of the DR1 was selected to be 10.2 and the dielectric constant $\varepsilon_{\mathrm{r} 2}$ of the DR2 was selected to be 2.2 .

\section{E. Design Procedure}

The design procedure of the proposed array is described in the following paragraphs.
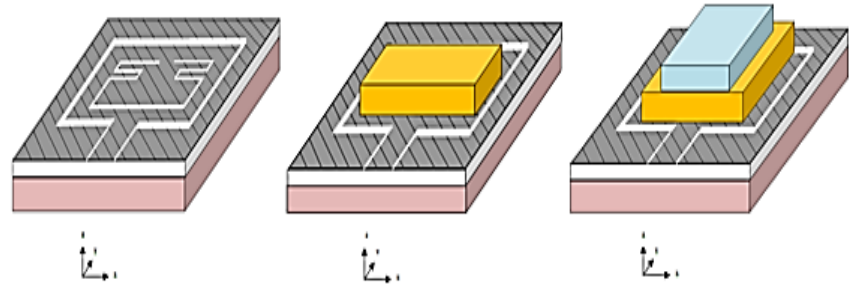

Fig. 14. Antenna design steps

In Step 1, the dimension of DR1 is first predicted using the simplified equations in [30], at $\mathrm{f}_{0}=60 \mathrm{GHz}$, and adjusted by 3D EM simulation. A meander slot feeding structure is designed to excite the dominant mode of DR1. In order to increase the bandwidth of the antenna, the slot feeding structure is designed as a radiator [31] at a resonant frequency that is close to that of DR1.

In Step 2, DR2 is also deliberated to resonate at $\mathrm{f}_{0}=60$ GHz. The placement and dimension of DR2 will not affect the field distribution inside DR1. This also implies that DR2 has no effect on the input impedance of the antenna then we have validated the observation through simulations.

\section{F. Simulation Results}

This section presents the simulated results of the meander slot antenna. CST has been used to simulate the antenna for several performance parameters.

It is evident in Figure 15 that the antenna adaptation increases with the number of DRs, but reaches a tray with three or more DRs. Considering the gain performance and the assembly difficulties; the compromised number of DRs is two or three for each on-chip antenna. In this paper, we will focus only on the two-DR configuration.

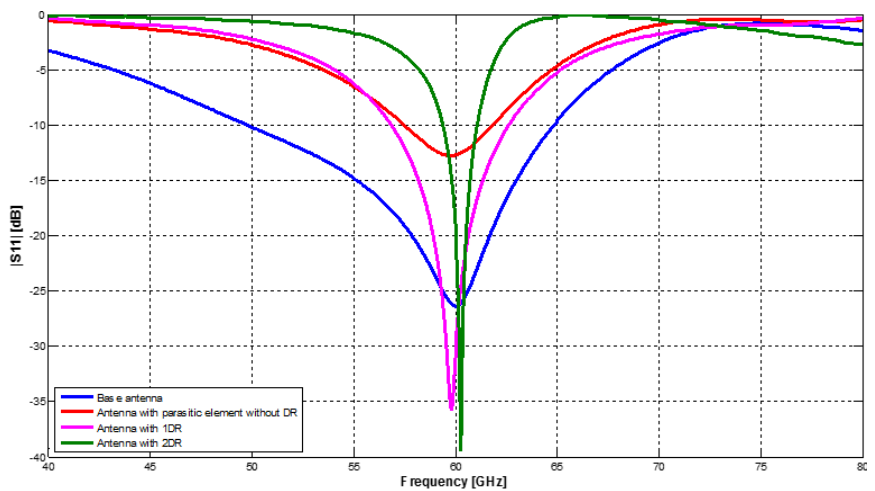

Fig. 15. Simulation results of the proposed antenna

The characteristics of the four antennas can be summarized as follows: according to the simulation results, we can see that the entire proposed antenna presents a resonance at $60 \mathrm{GHz}$. The main advantage of using dielectric resonator is the possibility of reducing the size of the antenna by more than $40 \%$ of based antenna.

The final structure have an interesting reflection coefficient simulation. We have validate our work with scattering bond graph methodology by obtaining the simulation shown on the Figure 16.

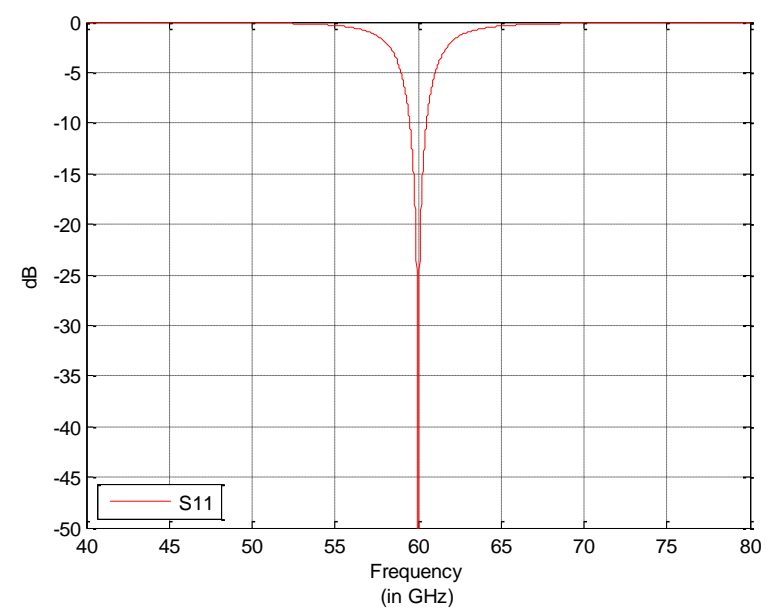

Fig. 16. Simulation of reflection coefficient of final antenna by Scattering Bond Graph methodology

Besides, the antenna is more adapted and the decreasing of the gain is little is from about $3 \mathrm{~dB}$ to $2.35 \mathrm{~dB}$. Antennas with multi-DRs are also investigated.

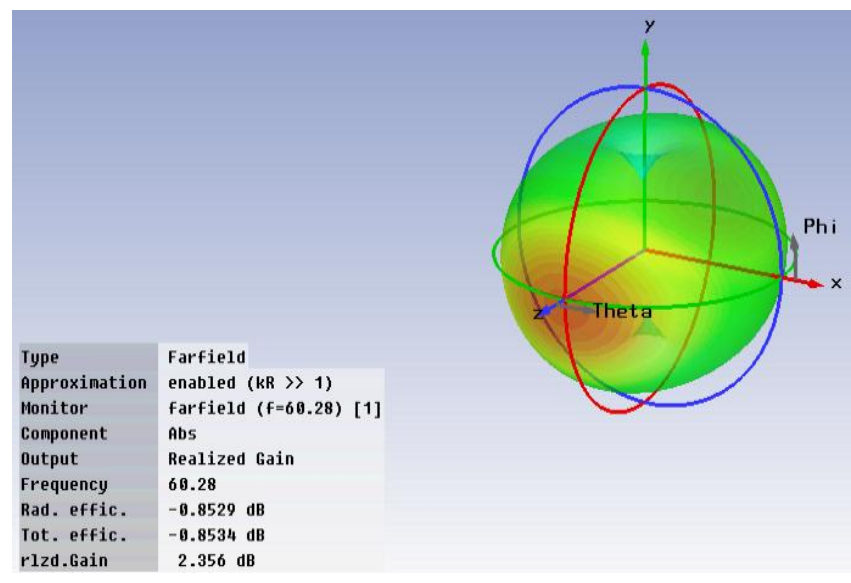

Fig. 17. Simulated gain of proposed antenna

\section{G. Comparative Study with a UHF Antenna Trade}

We are comparing our structure with the RFID antenna reader for Tag Product Company in terms of space and gain. Table 1 shows the study.

TABLE I. A Comparative Study Between the Studied ANTENNA AND THE BUSINESS ANTENNA

\begin{tabular}{|l|l|l|}
\hline & $\begin{array}{l}\text { Patch antenna } \\
\text { (Tag Product) }\end{array}$ & Proposed antenna \\
\hline Frequency $(\mathrm{GHz})$ & 0.869 & 60 \\
\hline Gain $(\mathrm{dB})$ & 6 & 2.35 \\
\hline Size $\left(\mathrm{L}^{*} \mathrm{~W}^{*} \mathrm{~h}\right)\left(\mathrm{mm}^{3}\right)$ & $260 * 260 * 36$ & $1.2 * 1.1 * 0.322$ \\
\hline Volume $\left(\mathrm{dm}^{3}\right)$ & 2.43 & 0.322 \\
\hline
\end{tabular}


From the table above, we found that:

- The basic structure of the dielectric resonator has a total volume of $0.322 \mathrm{dm}^{3}$ compared to patch antennas, which have a trade volume of $2.43 \mathrm{dm}^{3}$. We notice a significant miniaturization.

- The gain of the DRA is lower of 3dBi compared to the patch with Tag Product antenna, which seems logical to us because we used a high-permittivity dielectric resonator $\left(\varepsilon_{\mathrm{r}}=10.2\right)$.

\section{CONCLUSION}

A novel antenna for an RFID TAG was simulated and evaluated. The proposed antennas have a resonant frequency equal to $60 \mathrm{GHz}$ and it presents a very compact size and an acceptable gain.

In the first part, we presented the design of a patch antenna operating at $60 \mathrm{GHz}$ linearly polarized then we added in this structure the loop meander slot. This form allowed us to reduce antenna size by $38 \%$.

In the second part, we focused on designing a DRA in which the radiating element is formed by the arrangement of rectangular DR portions of different permittivity. The goal was to excite the two resonators to the desired frequency (60 $\mathrm{GHz}$ ).

The use of dielectric resonator was a very useful and fast tool to ameliorate the antenna. This work is one of the first steps in the millimeter wave identification (MMID) and we desire in the future to use our antenna in MMID TAG ChipLess.

The scattering bond graph methodology, used in this paper as a new design technique has shown its effectiveness in helping to understand and to interpret the studied antenna. This methodology can be used as a substitute for the iterative method since we can move from the first iteration to the last iteration without going through intermediate iterations because the scattering bond graph model can be simplified easily.

\section{ACKNOWLEDGMENT}

The preferred spelling of the word "acknowledgment" in America is without an "e" after the "g". Avoid the stilted expression "one of us (R. B. G.) thanks ...". Instead, try "R. B. G. thanks...". Put sponsor acknowledgments in the unnumbered footnote on the first page.

\section{REFERENCES}

[1] A. Lehto, J. Nummela, L. Ukkonen, L. Sydänheimo and M. Kivikoski,. Passive UHF RFID in paper industry: Challenges, benefits and the application environment. Transactions on Automation Science and Engineering, IEEE, 6(1), (2009), pp. 66-79.

[2] E. Abdulhadi, and A. Ramesh "Design and experimental evaluation of miniaturized monopole UHF RFID tag antennas." Antennas and Wireless Propagation Letters, IEEE 11 (2012): 248-251.

[3] P. Pursula, T. Vähä-Heikkilä, A. Müller, D. Neculoiu, G. Konstantinidis, A. Oja, J. Tuovinen, "Millimetre Wave Identification - new radio system for low power, high data rate and short range," IEEE Trans. on Microwave theory tech., vol. 56, no. 10, pp. 2221-2228, Oct. 2008.

[4] Lu, Kai, Kwok Wa Leung, and Yong Mei Pan. "Theory and experiment of the hollow rectangular dielectric resonator antenna." Antennas and Wireless Propagation Letters, IEEE 10 (2011): pp.631-634.
[5] Q. H. Lai, C. Fumeaux, W. Hong, and R. Vahldieck, "60 GHz aperture coupled dielectric resonator antennas fed by a half-mode substrate integrated waveguide," IEEE Trans. on AP, vol.58, no.6, pp.1856-1863, 2010.

[6] S. JMAL. 'Etude d'une méthodologie Bond Graph pour la modélisation des circuits hauts fréquences". Master thesis, faculty of sciences of Tunis, Tunisia. (2012)

[7] H. Taghouti and A. Mami. "How to Find Wave-Scattering Parameters from the Causal Bond Graph Model of a High Frequency Filter."American Journal of Applied Sciences (2010).

[8] R. MEHOUACHI, H. TAGHOUTI and MAMI, A., "Analysis of microstrip patch antenna array by using a new Bond Graph technology ". American Journal of Applied Sciences, 11 (8): 1436-1449, June, 2014

[9] Balanis, Constantine A. Antenna theory: analysis and design. Vol. 1. John Wiley \& Sons, 2005.

[10] T. Jang, J. Choi and S. Lim, "Compact Coplanar Waveguide (CPW)-Fed Zeroth-Order Resonant Antennas With Extended Bandwidth and High Efficiency on Vialess Single Layer", IEEE Transactions on Antennas and Propagation Vol 59, no. 2, pp. 363 - 372, Feb. 2011.

[11] S. Jmal, H. Taghouti, A. Mami, Design of a Ka Band Antenna by a New Methodology Based on Bond Graph Approach, International Journal on Communications Antenna and Propagation (IRECAP), 5(5), (2015) pp. 301-306.doi:http://dx.doi.org/10.15866/irecap.v5i5.7565

[12] H.Taghouti, , A. Mami, and S. Jmal, Nouvelle Technique de Modélisation et Simulation par Bond Graph: Applications aux Circuits Hauts Fréquences et Antennes Patch. (2014) Éditions universitaires européennes.

[13] B. Razavi, "Design of millimeter-wave CMOS radios: A tutorial," IEEE Transaction Circuits Systems. I, Reg. Papers, vol. 56, no. 1, pp. 4-16, Jan. 2009.

[14] Y. C. Ou and G. M. Rebeiz, "On-chip slot-ring and high-gain horn antennas for millimeter-wave wafer-scale silicon systems," IEEE Trans.Microwave Theory Tech., vol. 59, no. 8, pp. 1963-1972, Aug. 2011.

[15] S. JMAL, H. TAGHOUTI and A. MAMI, "Modeling and simulation of a patch antenna from its Bond Graph model". International Conference on Control, Decision and Information Technologies (CoDIT), 2014. IEEE, 2014. p. 609-614.

[16] S Jmal, H.Taghouti and A. Mami. "A new modeling and simulation methodology of a patch antenna by Bond Graph approach". International Conference on Electrical Engineering and Software Applications (ICEESA), 2013, pp. 1-6. IEEE.

[17] TK. Chen and GH. Huff," On the Constant Input Impedance of the Archimedean Spiral Antenna in Free-Space", Vol 62, no. 7, pp. 3869 3872, July 2014.

[18] J. J.Wang, , Y. P.Zhang, , K. M. Chua and Lu, A. C. W. "Circuit model of microstrip patch antenna on ceramic land grid array package for antenna-chip codesign of highly integrated RF transceivers". Antennas and Propagation, IEEE Transactions on, 53(12), 3877-3883. (2005).

[19] L. A Belov, S.M. Smolskiy, and V. N. Kochemasov, "Handbook of RF, Microwave, and Millimeter-wave Components". Artech House. (2012)

[20] I. J. Bahl. "Lumped elements for RF and microwave circuits". Artech house. (2003)

[21] K. C. Gupta, R. Garg, L. Bahl, and P. Bhartia. "Microstrip Lines and Slotlines". Second edition. Arthech House.

[22] KX. Wang and H. Wong, "A Circularly Polarized Antenna by Using Rotated-Stair Dielectric Resonator", IEEE Antennas and Wireless Propagation Letters, Vol 14, pp 787 - 790, 2015.

[23] X. Sheng Fang, K. Wa Leung "Linear-/Circular-Polarization Designs of Dual /Wide-Band Cylindrical Dielectric Resonator Antennas" IEEE Transactions On Antennas And Propagation, Vol. 60, no. 6, JUNE 2012.

[24] Y. Ding, K. Wa Leung and K. Man Luk, "Compact Circularly Polarized Dual-band Zonal-Slot/DRA Hybrid Antenna Without External Ground Plane", Antennas and Propagation, IEEE Transactions, Vol. 59, no. 6, pp. $2404-2409,2011$.

[25] S. Dhar, R. Ghatak, B. Gupta, D.R Poddar, "A Wideband Minkowski Fractal Dielectric Resonator Antenna "Antennas and Propagation, IEEE Transactions on", Vol 61, no. 6, pp. 2895 - 2903, 2013. 
[26] J. Avella Castiblanco, D. Seetharamdoo, M. Berbineau, M. Ney and F. Gallee, "Electromagnetic modeling and definition of antenna specifications and positions for radio system deployment in confined environments", IOP Conference Series: Materials Science and Engineering, 2013.

[27] D. H. Neil H. E. Weste, CMOS VLSI Design, 3rd ed. Addison Wesley, 2004.

[28] P. V. Bijumon, Y. Antar, A. P. Freundorfer, and M. Sayer, "Dielectric resonator antenna on silicon substrate for system on-chip applications," IEEE Trans.on AP, vol. 56, no. 11, pp. 3404-3410, Nov. 2008.
[29] M. R. Nezhad-Ahmadi, M. Fakharzadeh, B. Biglarbegian, and S. SafaviNaeini, "High-efficiency on-chip dielectric resonator antenna for mmwave transceivers," IEEE Transon AP, vol. 58, no. 10, pp. 3388-3392, Oct. 2010

[30] D. Hou, Y. Z. Xiong, W. Hong, W. L. Goh, and J. Chen, "Silicon-based On-chip Antenna Design for Millimeter wave/THz Applications," in Proc. IEEE Electrical Design of Advanced Package. Systems Symp, pp. 1-4, Dec. 2011.

[31] SY. Yang, M. Ng Mou Kehn, “A Bisected Miniaturized ZOR Antenna with Increased Bandwidth and Radiation Efficiency", IEEE Antennas and Wireless Propagation Letters, Vol. 12, pp. 159 - 162, 2013. 